\title{
Miranda
}

Revue pluridisciplinaire du monde anglophone /

Multidisciplinary peer-reviewed journal on the English-

speaking world

$17 \mid 2018$

Paysages et héritages de David Bowie

\section{Nancy Cunard, Parallaxe et autres poèmes. Hors-la-loi et Sublunaire}

\author{
Christine Dualé
}

\section{OpenEdition}

Journals

Édition électronique

URL : http://journals.openedition.org/miranda/15493

DOI : 10.4000/miranda. 15493

ISSN : 2108-6559

Éditeur

Université Toulouse - Jean Jaurès

Référence électronique

Christine Dualé, «Nancy Cunard, Parallaxe et autres poèmes. Hors-la-loi et Sublunaire », Miranda [En

ligne], 17 | 2018, mis en ligne le 02 octobre 2018, consulté le 16 février 2021. URL : http:// journals.openedition.org/miranda/15493; DOI : https://doi.org/10.4000/miranda.15493

Ce document a été généré automatiquement le 16 février 2021.

\section{(c) (i) () $\Theta$}

Miranda is licensed under a Creative Commons Attribution-NonCommercial-NoDerivatives 4.0 International License. 


\title{
Nancy Cunard, Parallaxe et autres poèmes. Hors-la-loi et Sublunaire
}

\author{
Christine Dualé
}

\section{RÉFÉRENCE}

Nancy Cunard, Parallaxe et autres poèmes. Hors-la-loi et Sublunaire. Traduction de Dorothée Zumstein. Les Nouvelles éditions Jean-Michel Place. Or-la-loi dirigé par Cyrille Zola-Place (Paris : Les Nouvelles éditions, 2016), 125 p, ISBN : 9782858939923

1 " On connaît en France Nancy Cunard. Elle y a passé la majeure partie de sa vie et plus tard on ne pourra faire, sans parler d'elle, l'histoire intellectuelle d'une part de ce siècle ». Ainsi est évoquée Nancy Cunard dans Les lettres françaises (1960) de Louis Aragon, qui prédit le rôle et l'empreinte indélébiles de cette femme singulière dans l'histoire intellectuelle et poétique du siècle dernier. Pourtant, qui connaitt la poésie de Nancy Cunard, son action intellectuelle, artistique et politique, son engagement résolument moderne dans la première moitié du vingtième siècle ? "L'histoire intellectuelle du XXe siècle s'est faite sans elle » (8).

Dirigé par Cyrille Zola-Place, Parallaxe et autres poèmes propose de faire renaître l'œuvre poétique (écrite entre 1921 et 1925) de Nancy Cunard mais aussi de la diffuser largement puisqu'il s'agit d'une édition bilingue, les poèmes ayant été traduits par Dorothée Zumstein.

3 Poète, collectionneuse d'art africain, modèle pour Brancusi et Man Ray, journaliste, femme libre, icône de l'entre-deux-guerres, aristocrate anglaise née en 1896 et installée à Paris en 1920, symbole de l'avant-garde anglo-saxonne et française, Nancy Cunard évolue parmi les intellectuels avant-gardistes, surréalistes et dadaïstes et met sa fortune au service des artistes et intellectuels. « Sa beauté, ses audaces, son goût pour l'excès, son appétit pour les hommes et pour les femmes avaient provoqué une multitude de scandales qui éclatèrent à Londres, à New York et à Paris pendant l'entredeux-guerres »(20), comme l'explique Alexandra Lapierre dans Avec toute ma colère 
(2018). Nancy Cunard fut le symbole de la liberté féminine dans une société dominée par le poids des traditions. Son amie Iris Tree, poète et actrice, proche de l'avant-garde anglo-saxonne (citée par Sarah Frioux-Salgas dans son article «Introduction «L'Atlantique noir » de Nancy Cunard, Negro Anthology, 1931-1934 » (Gradhiva [En ligne], 19|2014, URL: http://journals.openedition.org/gradhiva/2771) témoigne de cette «force agissante » (12) et résume parfaitement le parcours et la vie tourbillonnante de Nancy Cunard :

[...] À ce moment-là, nous étions des bandits, n'hésitant pas à nous maquiller avec de la craie blanche sur le visage et du rouge à lèvres écarlate, fumant des cigarettes parmi des fêtards choisis par nous-mêmes [...] Nous étions de vrais caméléons. Nous passions de Meredith à Proust et à Dostoïevski, goûtions à l'absinthe avec Baudelaire et Oscar Wilde, [...] nous nous laissions assombrir par le pessimisme nihiliste, [...] inspirées par le jeune Rupert Brooke, T.S. Eliot, Yeats, D.H. Laurence, secouées par Blast de Wyndham Lewis, [...] la « signifiant form », [...], les sculptures d'Epstein, la musique de Stravinsky, les premiers ballets russes et le jazz américain " (Ford 1968: 19, traduit par Sarah Frioux-Salgas in, «Introduction «L'Atlantique noir » de Nancy Cunard, Negro Anthology, 1931-1934»).

4 Cet ouvrage, agrémenté de dessins de Sylvie Bonnot (tirés de la série New Izu Hotel), présente les poèmes que Nancy Cunard écrivit entre vingt et trente ans et dont l'accueil fut plus ou moins mitigé. Hors-la-loi (1921), notamment, fut vivement critiqué par Ezra Pound qui appelait à une langue plus épurée et sobre avant de devenir le chef de file de l'imagisme. Mais Nancy Cunard ne tiendra pas compte de ses reproches et publiera Sublunaire en 1923. Parallaxe paraît en 1925 et c'est sur ce long poème, " qui répond aux critères formels de la modernité » (9) et par lequel Nancy Cunard cherche à s'affranchir des règles d'écriture de l'Angleterre victorienne, que s'ouvre l'ouvrage. Elle écrit pourtant dans Parallax: « [...] Ah no - the new cannot put out the old - / Though I clutch on the new I shall not shuffle off the old, / Wrapped, folded together, / The new burns, ripens in the known, / Folded, growing together - / Yes - (even to paradox) / Have I not loved you better, loving again?" (Parallax 42).

5 Les poèmes de Nancy Cunard illustrent ce dont elle fut le témoin, reflètent une écriture poétique kaléidoscopique et insistent sur l'importance de la mort avant la vie: «The death-before-life, the atom in the womb / Preparing - snarled embryos, pinched / By once-roseate poisons. / (Frail brown / Pre-natal dust, what life is it you missed? » (44). Témoin de la Première Guerre mondiale à Londres, une ville qu'elle n'aimait pas, une fois installée à Paris elle écrira la mémoire de cette guerre, de sa jeunesse et de ce mouvement perpétuel: "London - Youth and heart-break / Growing from ashes. / The war dirges/ Burning, reverberate-burning/ Now far away, sea-echoed, now in the sense, / Taste, mind, uneasy quest of what I am - / London, the hideous wall, the jail of what I am [...]" (Parallax 26).

Elle laisse des poèmes qui produisent du sens, interrogent le sens. "La parallaxe ne change pas seulement l'apparence de l'objet, mais aussi l'être du sujet. Nous touchons là à la singularité et à l'actualité de sa poésie, qui ne se réduit pas à un formalisme mais produit, par la discontinuité de la langue liée aux ruptures de syntaxe, un espace poétique singulier. L'effet de collage cher à une modernité incarnée par Eliot est alors dépassé par des chevauchements syntaxiques [...]» (12).

7 Proche du Bloosmbury group qui regroupe des écrivains et artistes (dont Leonard et Virginia Woolf), prônant la théorie de la « forme signifiante » (c'est-à-dire l'importance de la forme sur le contenu), Nancy Cunard «s'inscrit dans les mouvements d'idées 
modernistes de l'histoire intellectuelle anglaise du début du xxe siècle » (Frioux-Salgas op.cit.). Les époux Woolf donneront d'ailleurs sa chance au recueil Parallax en le publiant dans leur maison d'édition Hogarth Press.

8 Les premiers poèmes de Nancy Cunard sont publiés en 1916 mais ce sont les recueils écrits entre 1921 et 1925 qui sont proposés ici : Parallax/ Parallaxe (1925), Southern Sheriff (extrait de l'Anthologie Noire, 1934), Outlaws / Hors-la-loi (1921), Sublunary/ Sublunaire (1923). Écrits pour la plupart au sortir de la Première Guerre mondiale, ces poèmes sont un manifeste de la modernité anglo-saxonne qui se veut un mouvement anti-victorien et anti-romantique. Nancy Cunard veut s'affranchir de la tradition et défend une nouvelle liberté, de mœurs, mais aussi d'écriture, appelant à la libération des singularités. Paris, où elle s'installe en 1920, deviendra l'espace de cette liberté tant recherchée.

9 Les poèmes du recueil Outlaws/Hors-la-loi, écrits en versets libres, témoignent de son engagement politique et de sa lutte contre le racisme, elle qui couvrit la guerre d'Espagne aux côtés des Républicains. Quant au poème Southern Sheriff, extrait de l'Anthologie Noire de 1934, il permet de rappeler l'ouvrage pour lequel Nancy Cunard est plus connue mais aussi ses activités d'éditrice car elle établit sa propre maison d'édition, Hours Press: "C'est [...] avec Aragon qu'elle fonde, en 1928, sa maison d'édition, Hours Press, qui devait défendre "l'innovation et une nouvelle vision des choses » (Cunard 1969) et publier de la poésie expérimentale [...] Elle découvre le métier d'éditeur-imprimeur avec Aragon, mais c'est surtout avec son nouveau compagnon Henry Crowder, pianiste africain-américain rencontré à Venise, l'été 1928, qu'elle le pratique pendant quatre ans (Crowder et Speck 1987 ; Barnett 2007). Elle édite vingttrois ouvrages en anglais et en tirages limités, dont le premier texte publié de Samuel Beckett, Whoroscope 30, et un des volumes des Cantos de Pound "(Fioux-Salgas, op. cit.).

Southern Sheriff s'élève contre le racisme en général et le racisme anti-noir aux ÉtatsUnis: " White folks don't kill each other in the South / Ho no, not with so many niggers around. / It's the wrong end of the stick you got, englishman [...]" (Southern Sheriff 58). Engagée dans la lutte contre le racisme et confrontée très tôt aux questions coloniales, Nancy Cunard conçoit la Negro Anthology comme une riposte antiraciste et anticolonialiste. Après sa rencontre avec le pianiste de jazz Henry Crowder, Nancy Cunard, qui découvrit la vie des Noirs américains et le racisme à travers les journaux qu'il lui faisait lire (Crisis et Liberator), devint militante pour la cause noire et l'égalité raciale et décida de se consacrer à la publication de cette anthologie, en rassemblant les poésies et les essais d'auteurs noirs américains mais aussi ceux d'auteurs des Caraïbes et d'Europe. Elle réussit à décloisonner les genres à travers ce qui fut le premier exemple de collaboration entre Blancs et Noirs. Très controversée et peu aidée par la presse, Nancy Cunard n'abandonna jamais son objectif et publia Negro: An Anthology en 1934. Cet ouvrage fut le premier à exploiter la voix des Noirs en proposant des articles et des travaux sur leur condition en Afrique, en Amérique du Nord et du Sud, en Europe et dans les Caraïbes et en mettant en avant la plupart de leurs modes d'expression comme la musique, la poésie, les arts et l'histoire. En dépit de ses 870 pages, son ouvrage fut critiqué pour ressembler davantage à un journal de voyage relatant son opinion anecdotique qu'à un véritable document politique et ethnographique :

«Le contexte de création de Negro [...] explique l'originalité de cet ouvrage mais aussi les contradictions intellectuelles et politiques [...], et l'incohérence apparente de son sommaire divisé en sept sections géographiques et thématiques: America, Negro stars, Music, Poetry, West Indies and South America, Europe, Africa. Negro 
réunit des textes inédits et compile de nombreux articles, poèmes ou extraits d'ouvrages déjà publiés. On y trouve également un très grand nombre de photographies. L'intérêt de ce corpus iconographique, outre sa taille, réside dans les légendes très précises ou les commentaires personnels de l'éditrice qui accompagnent systématiquement les images. Cette approche souvent subjective participe à la singularité de cette publication non universitaire et totalement artisanale. [...] Negro Anthology s'inscrit aussi dans le courant du documentaire social américain » (Fioux-Salgas, op. cit.).

L'ambition de Nancy Cunard avec cet ouvrage ne fut pas comprise du public, elle qui voyait dans la défense de l'africanité un moyen de régénérer la culture occidentale jugée stérile. En défendant le maintien du primitivisme noir, elle pensait pouvoir préserver l'authenticité culturelle qu'elle réclamait mais elle proposa finalement une vision idéalisée et stéréotypée. Toutefois, Negro Anthology est « une synthèse majeure unique et originale de la diversité des discours scientifiques, politiques et culturels des Noirs et sur les Noirs dans les années 1930 » (ibid). Ce sont ces aspects-là, son infatigable dénonciation des injustices, ainsi que ce travail d'émancipation singulière qu'il faut retenir de Nancy Cunard.

Femme libre et libérée avant l'heure, fascinée par l'Afrique et obsédée par la cause noire, « l'égalité des races, l'égalité des sexes, l'égalité des classes » (Cunard in Lapierre, 27), Nancy Cunard fut sans nul doute, pour le monde intellectuel parisien de l'époque, une grande influence. Le rôle important qu'elle joua sur la scène intellectuelle parisienne est malheureusement peu évoqué, voire méconnu. Cette édition bilingue de Cyrille Zola-Place qui « propose de faire renaitre son œuvre [...] [et de] rouvrir une voie à une pratique poétique dont le verso est singulièrement politique » (8), invite surtout à découvrir ou à redécouvrir la sensibilité et l'écriture d'une femme de contradictions, résolument moderne, qui décloisonna les genres et dont «l'action s'est déroulée sur tous les champs de bataille, politique et poétique » (124).

\section{INDEX}

Keywords : acting strength, alterity, avant-guard, Bloosmbury group, Hours Press, London, London counter-culture, female freedom, femininity, icon, imagism, modernism, Paris, roaring twenties, signifiant form, singularity

Mots-clés : altérité, Années folles, avant-garde, contre-culture londonienne, groupe de Bloosmbury, féminité, force agissante, forme signifiante, Hours Press, icône, imagisme, liberté féminine, Londres, modernisme, Paris, singularité

\section{AUTEURS}

\section{CHRISTINE DUALÉ}

Maître de conférences HDR

Université Toulouse Capitole

christine.duale@ut-capitole.fr 